\title{
Knockdown of ST7-AS1 inhibits migration, invasion, cell cycle progression and induces apoptosis of gastric cancer
}

\author{
SHUANG CAI, YANG WENG, PENGLIANG LIU and FENG MIAO
}

The Fourth Affiliated Hospital of China Medical University, Shenyang, Liaoning 110032, P.R. China

Received September 19, 2019; Accepted November 14, 2019

DOI: $10.3892 / 01.2019 .11145$

\begin{abstract}
Role of ST7-AS1 in the malignant progression of gastric cancer (GC) and its molecular mechanisms were investigated. ST7-AS1 level in GC tissues and matched normal tissues was determined by quantitative real-time polymerase chain reaction (qRT-PCR). Its level in GC patients presenting different tumor stages and tumor sizes was determined. Subsequently, ST7-AS1 level in epithelial cells of gastric mucosa and GC cell lines was examined. Cellular behavior of GC cells, including viability, apoptosis, migration, invasion and cell cycle, influenced by ST7-AS1 was evaluated. The interaction between ST7-AS1 and EZH2 was assessed by RNA immunoprecipitation (RIP) assay. The involvement of EZH2 in the progression of GC mediated by ST7-AS1 was identified. ST7-AS1 was upregulated in GC tissues and cell lines. Its level was positively correlated to tumor stage and tumor size of GC. Knockdown of ST7-AS1 attenuated proliferative, migratory and invasive abilities, arrested cell cycle progression and induced apoptosis of GC cells. EZH2 was identified to interact with ST7-AS1, which attenuated the regulatory effects of ST7-AS1 on migratory and invasive abilities of GC cells. Upregulated ST7-AS1 in GC accelerated proliferation, migration and invasion, and inhibited apoptosis, thus aggravating the progression of GC.
\end{abstract}

\section{Introduction}

Gastric cancer (GC) is a malignant tumor originating from the gastric mucosa. It ranks the first in tumor incidence of Chinese population. Regional difference is observed in the incidence of GC. The northwest and eastern coastal areas of China are high incidence regions of GC (1). GC mainly affects people over 50 years, with the male-female ratio of $2: 1$. Recently, the incidence of GC becomes younger owing to changes in environmental and dietary habits, overloaded working and

Correspondence to: Dr Feng Miao, The Fourth Affiliated Hospital of China Medical University, 4 Chongshan Road, Huanggu, Shenyang, Liaoning 110032, P.R. China

E-mail: aefra602@163.com

Key words: GC, ST7-AS1, EZH2, progression increased infection rate of Helicobacter pylori (2). GC occurs in any part of the stomach, and more than half of GC cases structurally involve the gastric antrum (3). Based on the pathological classification, the majority of GC belongs to adenocarcinoma. Early-stage symptoms of GC are atypical, manifesting as similar symptoms of gastritis and gastric ulcer, such as upper abdominal discomfort and hernia (4). Unfortunately, detective rate of early-stage GC is low, leading to a poor prognosis of GC patients (5). The overall survival of $\mathrm{GC}$ remains at $20 \%$, which is a global health problem (6). Advanced GC has an extreme poor prognosis, with $<15 \%$ of 5-year survival (7). Development of effective and sensitive hallmarks for GC contributes to improving the clinical outcome of the affected (8).

Long non-coding RNAs (lncRNAs) are transcribed by RNA polymerase II with $>200$ nucleotides in length. They are a research focus on tumor-targeted therapy (9). IncRNAs are dysregulated in tumors, and mediate oncogenes or tumor-suppressor genes to further influence tumor progression (10). They exert diverse functions in regulating cellular behavior (11). In tumor biology, IncRNAs have been widely explored since they are capable of regulating drug-resistance and malignant phenotypes of tumor cells $(12,13)$. Tumor-related lncRNAs may be promising targets applied in tumor detection (14).

EZH2 encodes a histone lysine N-methyltransferase that is involved in DNA methylation to inhibit transcription of other genes. EZH2 also methylates H3K27me3 (15). The methylation activity of EZH2 promotes heterochromatinization and thus silences downstream genes (16). Mutation or overexpression of EZH2 is associated with multiple types of cancers (17-21). Abnormally activated EZH2 can inhibit expression of tumor-suppressor genes. Therefore, inhibition of EZH2 activity is able to alleviate tumor growth (22).

This study explored the biological function of lncRNA ST7-AS1 in the malignant progression of GC. The potential interaction between ST7-AS1 and EZH2 was investigated, which may provide new directions for developing therapeutic strategies for GC.

\section{Patients and methods}

Subjects. GC tissues and matched adjacent normal tissues were surgically harvested from GC patients in The Fourth Affiliated Hospital of China Medical University (Shenyang, 
China) from April 2016 to October 2018. Resected samples were placed into liquid nitrogen until analyses. Enrolled GC patients were pathologically diagnosed and had no medical history of other malignancies. This study was approved by the Ethics Committee of The Fourth Affiliated Hospital of China Medical University and informed consent was received from each subject.

Cell culture and transfection. Epithelial cells of gastric mucosa(GES-1) and GC cell lines (AGS,MG803 andSGC-7901) provided by American Type Culture Collection (ATCC) were cultured in Roswell Park Memorial Institute-1640 (RPMI-1640) containing 10\% fetal bovine serum (FBS) (both from HyClone) and $1 \%$ penicillin-streptomycin in a $5 \% \mathrm{CO}_{2}$ incubator at $37^{\circ} \mathrm{C}$. Prior to transfection, cells were seeded in a 6 -well plate with $1 \times 10^{4}$ cells/well. Serum-free medium $(1.5 \mathrm{ml})$ and $0.5 \mathrm{ml}$ of Lipofectamine ${ }^{\mathrm{TM}} 2000$ (Invitrogen; Thermo Fisher Scientific, Inc.) containing transfection vectors were mixed. At $75-85 \%$ confluence, $2.0 \mathrm{ml}$ of transfection mixture was applied in each well. Complete medium was replaced 4-6 h later.

Western blot analysis. Total protein was extracted from cells or tissues using radioimmunoprecipitation assay (RIPA) and loaded for electrophoresis (Beyotime). After transferring on a polyvinylidene fluoride (PVDF) membranes (Millipore), it was blocked in 5\% skim milk for $2 \mathrm{~h}$, incubated with primary antibodies at $4^{\circ} \mathrm{C}$ overnight and secondary antibodies for $2 \mathrm{~h}$. Bands were exposed by electrochemiluminescence (ECL) and analyzed by Image Software (NIH).

RNA extraction and quantitative real-time polymerase chain reaction ( $q R T-P C R)$. RNA extraction was performed using TRIzol method (Invitrogen; Thermo Fisher Scientific, Inc.). The extracted RNA was quantified and reverse transcribed into complementary deoxyribose nucleic acid (cDNA), followed by PCR using SYBR-Green method (Takara). QRT-PCR was performed at $94^{\circ} \mathrm{C}$ for $5 \mathrm{~min}$, and 40 cycles at $94^{\circ} \mathrm{C}$ for $30 \mathrm{sec}$, $55^{\circ} \mathrm{C}$ for $30 \mathrm{sec}$ and $72^{\circ} \mathrm{C}$ for $90 \mathrm{sec}$.

Cell counting kit-8 (CCK-8) assay. Cells were seeded in a 96-well plate with $2 \times 10^{3}$ cells/well. Absorbance (A) at $450 \mathrm{~nm}$ was recorded at the appointed time points using the CCK- 8 kit (Dojindo Laboratories) for depicting the viability curve.

Apoptosis determination. Apoptotic rate in GC cells was determined through calculating caspase-3 activity using a relevant commercial kit (Beyotime).

Flow cytometry. Cells were fixed in $75 \%$ ethanol at $4^{\circ} \mathrm{C}$ overnight, and washed with phosphate-buffered saline (PBS) twice. After incubation with RNase A at $37^{\circ} \mathrm{C}$ for $30 \mathrm{~min}$, cells were dyed with propidium iodide (PI). Cell cycle distribution was finally analyzed by FACSCalibur flow cytometry (BD Biosciences).

RNA immunoprecipitation (RIP). Cells were treated according to the procedures of Millipore Magna RIP ${ }^{\mathrm{TM}}$ RNA-Binding Protein Immunoprecipitation Kit (Millipore). Cell lysate was incubated with anti-EZH2 or IgG antibody at $4^{\circ} \mathrm{C}$ for $6 \mathrm{~h}$.
A protein-RNA complex was captured and digested with $0.5 \mathrm{mg} / \mathrm{ml}$ proteinase $\mathrm{K}$ containing $0.1 \%$ sodium dodecyl sulphate (SDS) to extract RNA. The magnetic beads were repeatedly washed with RIP washing buffer to remove non-specific adsorption as much as possible. Finally, the extracted RNA was subjected to mRNA level determination using qRT-PCR.

Transwell assay. Fifty microliters of FN $(100 \mu \mathrm{g} / \mathrm{ml})$ was coated in the bottom of Transwell chambers. Cell density was adjusted to $1 \times 10^{6} / \mathrm{ml}$. One hundred microliters of suspension was applied to the upper chamber of Transwell chambers (Millipore) pre-coated with $100 \mu \mathrm{l}$ of diluted Matrigel (BD Biosciences). Into the lower chamber, $600 \mu 1$ of medium containing $10 \%$ FBS was applied. After $48 \mathrm{~h}$ of incubation, invasive cells were fixed in methanol for $30 \mathrm{~min}$ and dyed with $0.1 \%$ crystal violet for $10 \mathrm{~min}$. Invasive cells were captured and counted in 6 randomly selected fields per sample. Migration assay was similarly performed except for Matrigel pre-coating.

Statistical analysis. Statistical Product and Service Solutions (SPSS) 19.0 software (IBM Corp.) was used for data analyses. Data were expressed as mean \pm standard deviation. Intergroup differences were analyzed by the t-test. $\mathrm{P}<0.05$ was considered statistically significant.

\section{Results}

Upregulation of ST7-AS1 in GC. Expression pattern of ST7-AS1 in GC tissues was examined. As qRT-PCR revealed, ST7-AS1 was upregulated in GC tissues relative to normal ones (Fig. 1A). Moreover, ST7-AS1 level was higher in GC tissues of $>5 \mathrm{~cm}$ in size than those $\leq 5 \mathrm{~cm}$ (Fig. 1B). Higher level of ST7-AS1 was observed in GC patients with stage III-IV compared with those with stage I-II (Fig. 1C). It indicated that ST7-AS1 was involved in the progression of GC.

Knockdown of ST7-AS1 suppresses viability, arrests cell cycle and induces apoptosis of GC. Compared with epithelial cells of gastric mucosa, ST7-AS1 was upregulated in GC cells, especially in AGS and SGC-7901 cells (Fig. 2A). Three transfection vectors of sh-ST7-AS1 (sh-ST7-AS1 1\#, sh-ST7-AS1 2\# and sh-ST7-AS1 3\#) were tested for their transfection efficacy. QRT-PCR data revealed pronounced transfection efficacy in the former two vectors (Fig. 2B). In AGS and SGC-7901 cells transfected with sh-ST7-AS1 1\# or sh-ST7-AS1 2\#, the viability greatly decreased compared with controls (Fig. 2C and D). Apoptotic rate was elevated by transfection of sh-ST7-AS1 1\# or sh-ST7-AS1 2\# (Fig. 2E). Moreover, cell ratio in G0/G1 phase was enhanced after transfection of sh-ST7-AS1 1\# or sh-ST7-AS1 2\# in GC cells, indicating arrested cell cycle progression (Fig. 2F).

Knockdown of ST7-AS1 suppressed migratory and invasive abilities of GC. After transfection of sh-ST7-AS1 1\# or sh-ST7-AS1 2\# in AGS and SGC-7901 cells, Transwell assay illustrated attenuated migratory and invasive abilities relative to controls (Fig. 3). 
A $\overline{\mathrm{s}}$

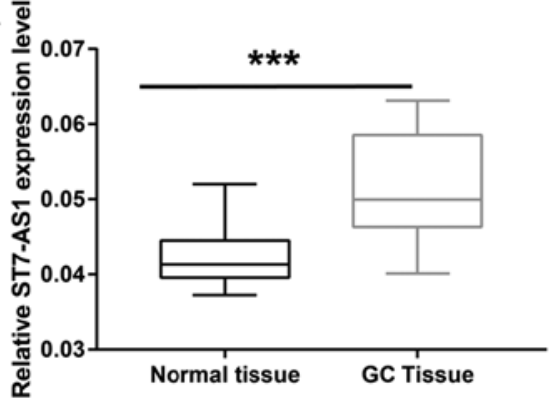

B -

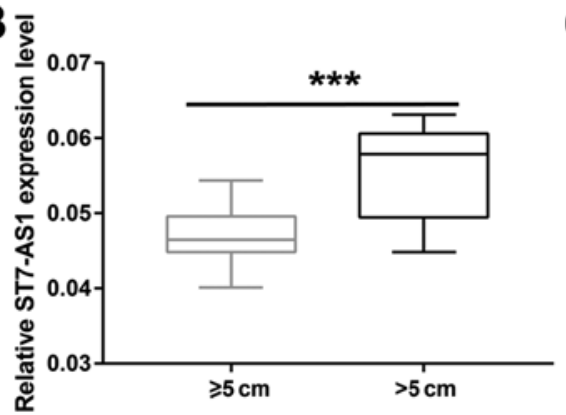

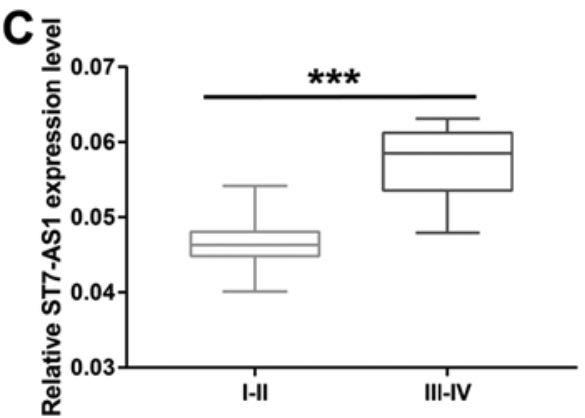

Figure 1. Upregulation of ST7-AS1 in GC. (A) Relative level of ST7-AS1 in GC tissues and matched normal tissues. (B) Relative level of ST7-AS1 in GC tissues $>5 \mathrm{~cm}$ in size and those $\leq 5 \mathrm{~cm}$. (C) Relative level of ST7-AS1 in GC patients in stage II-IV and stage I-II. ${ }^{* * *} \mathrm{P}<0.001$.

A

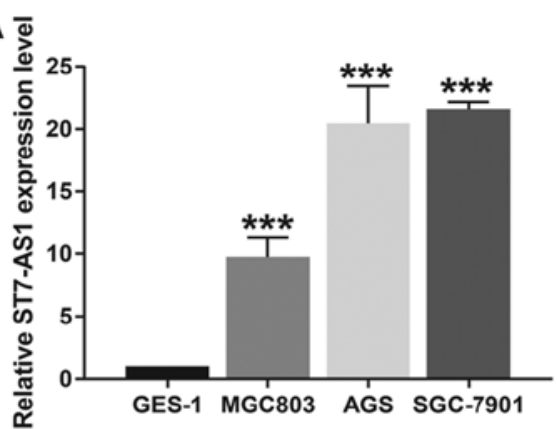

D

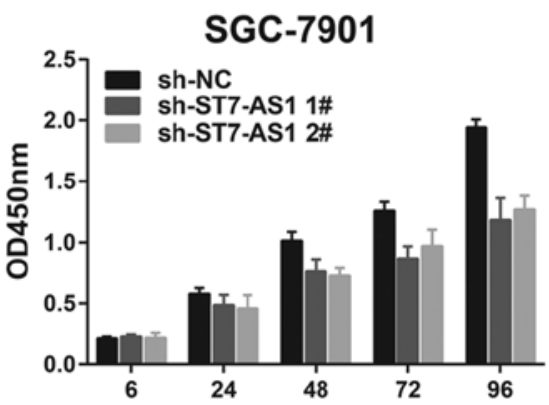

B
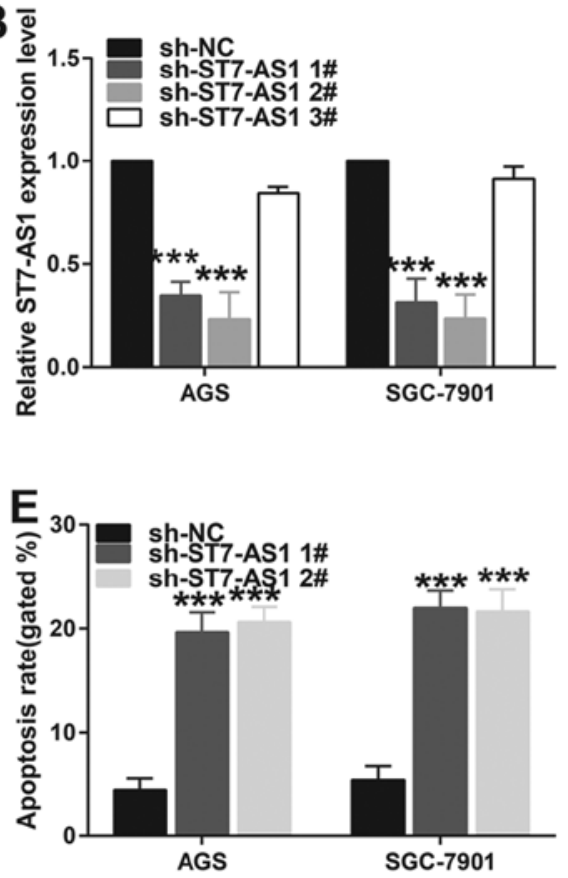

C

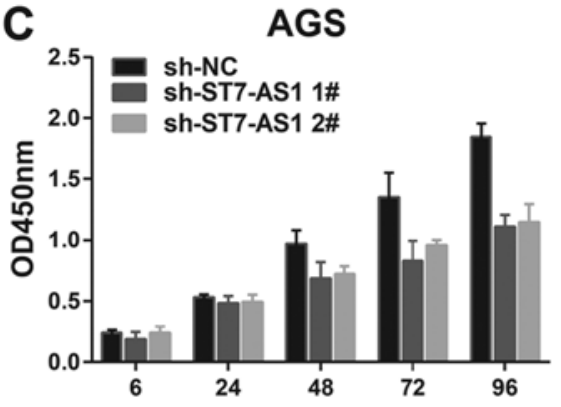

F

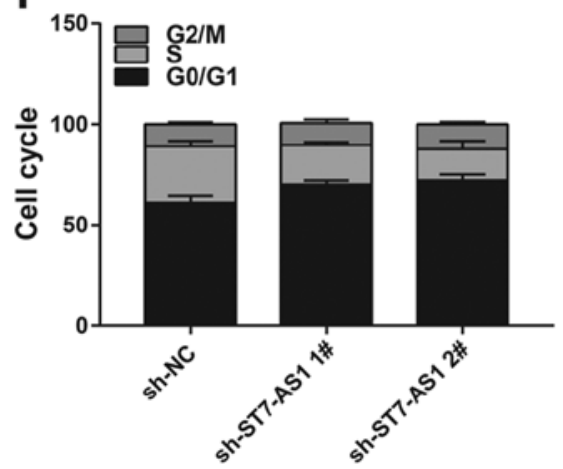

Figure 2. Knockdown of ST7-AS1 suppresses viability, arrests cell cycle and induces apoptosis of GC. (A) Relative level of ST7-AS1 in epithelial cells of gastric mucosa (GES-1) and GC cell lines (AGS, MG803 and SGC-7901). (B) Transfection efficacy of sh-ST7-AS1 1\#, sh-ST7-AS1 2\# and sh-ST7-AS1 3\# in AGS and SGC-7901 cells. (C) CCK-8 showed viability in AGS cells transfected with sh-NC, sh-ST7-AS1 1\# or sh-ST7-AS1 2\#. (D) CCK-8 showed viability in SGC-7901 cells transfected with sh-NC, sh-ST7-AS1 1\# or sh-ST7-AS1 2\#. (E) Apoptotic rate in AGS and SGC-7901 cells transfected with sh-NC, sh-ST7-AS1 1\# or sh-ST7-AS1 2\#. (F) Cell cycle distribution in AGS and SGC-7901 cells transfected with sh-NC, sh-ST7-AS11\# or sh-ST7-AS1 2\#. CCK-8, cell counting kit-8; GC, gastric cancer. ${ }^{* * *} \mathrm{P}<0.001$.

ST7-AS1 mediats cellular behavior of GC via interacting with EZH2. To uncover the molecular mechanism of ST7-AS1 in regulating cellular behavior of GC, RIP assay was conducted to assess the potential interaction between ST7-AS1 and EZH2.ST7-AS1 was greatly enriched in anti-EZH2 antibody relative to control $\mathrm{IgG}$, verifying the interaction between ST7-AS1 and EZH2 (Fig. 4A). Transfection of sh-EZH2 markedly downregulated protein level of EZH2, presenting an effective transfection efficacy in GC cells (Fig. 4B). Interestingly, the attenuated migratory and invasive abilities of GC cells transfected with sh-ST7-AS1 1\# were further inhibited by co-transfection of sh-EZH2 (Fig. 4C). It is indicated that ST7-AS1 accelerated GC cells to migrate and invade via interacting with $\mathrm{EZH} 2$.

\section{Discussion}

GC is a common malignancy worldwide, especially in China (23). Recently, identification of novel therapeutic hallmarks of GC have been widely conducted (24). GC is characterized by infinitely excessive proliferation and growth of tumor cells (25). Oncogene activation and tumor-suppressor gene inactivation are the main causes of tumorigenesis (26). Traditional treatments for GC include surgery, chemotherapy and radiotherapy. These therapeutic strategies destroy normal tissues and cells while destroying tumor tissues (27). In addition, the development of chemotherapy-resistance markedly limits the therapeutic efficacy of GC (28). Hence, it is urgent to search for effective targets for GC treatment. 

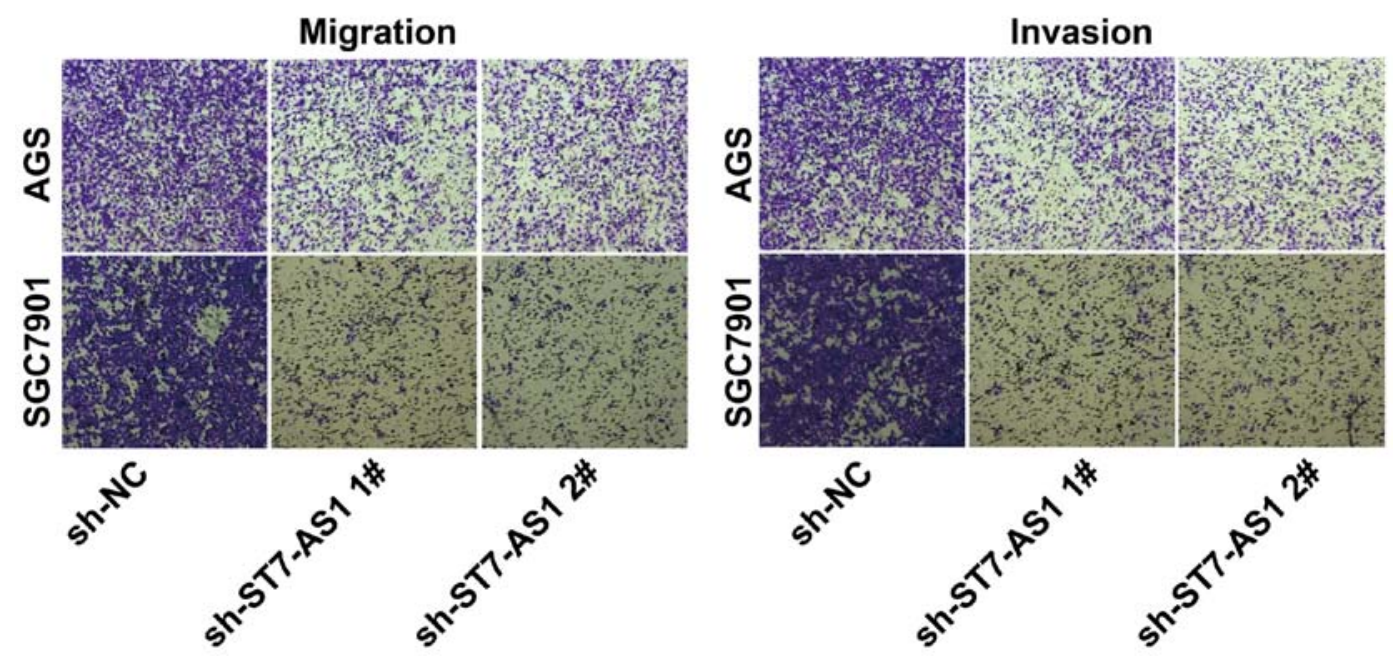

Figure 3. Knockdown of ST7-AS1 suppressed migratory and invasive abilities of GC. Transwell assay showed migration (left) and invasion (right) in AGS and SGC-7901 cells transfected with sh-NC, sh-ST7-AS1 1\# or sh-ST7-AS1 2\#. GC, gastric cancer; AGS, GC cell lines.

A

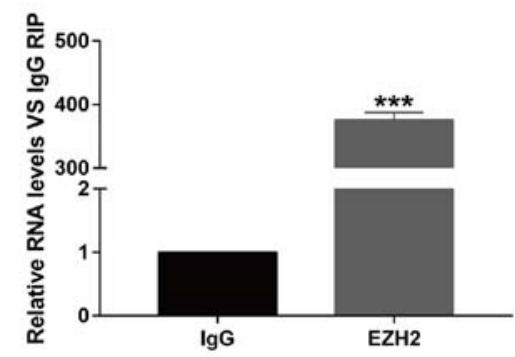

C
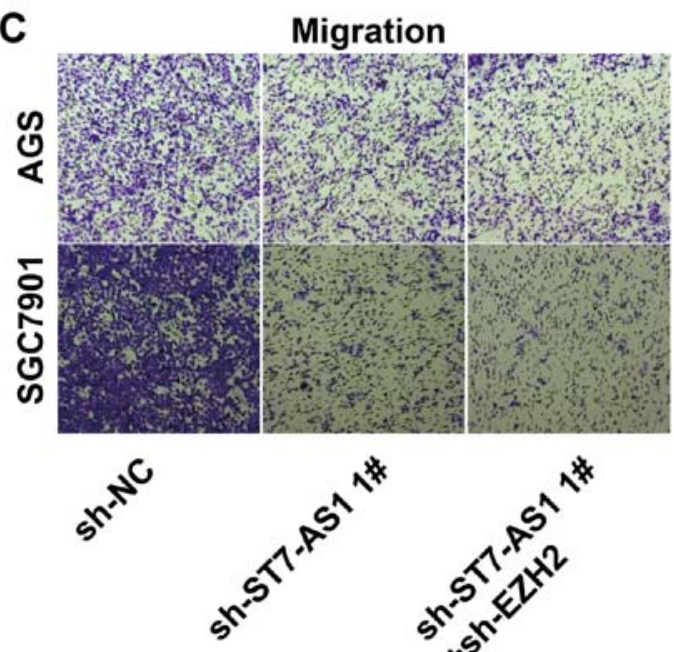

B
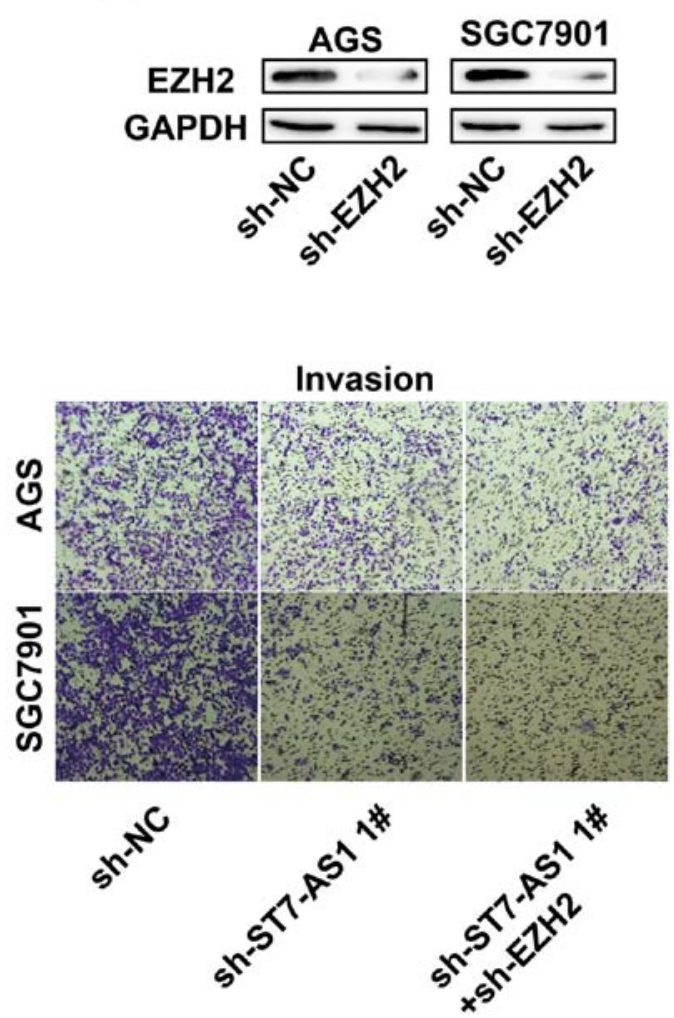

Figure 4. ST7-AS1 mediated cellular behavior of GC via interacting with EZH2. (A) RIP assay of the enrichment of ST7-AS1 in anti-IgG and anti-EZH2. (B) Transfection efficacy of sh-EZH2 in AGS and SGC-7901 cells. (C) Transwell assay of migration and invasion in AGS and SGC-7901 cells transfected with sh-NC, sh-ST7-AS1 1\# orsh-ST7-AS1 1\#+sh-EZH2. GC, gastric cancer; AGS, GC cell lines; RIP, RNA immunoprecipitation. ${ }^{* * *} \mathrm{P}<0.001$.

lncRNAs barely encode proteins, but mediate gene expression at multiple levels $(29,30)$. Increasing evidence has proven the role of IncRNAs in tumor progression (31). They are capable of mediating epigenetic regulation and cellular behavior (32). IncRNA ST7-AS1 is a newly discovered one located on 7q31.2 (33). In this study, ST7-AS1 was upregulated in GC tissues and cell lines. ST7-AS1 level was higher in GC patients with worse tumor stage and larger tumor size, indicating its carcinogenic role in GC. Moreover, knockdown of ST7-AS1 attenuated proliferative, migratory and invasive abilities, arrested cell cycle and induced apoptosis of GC cells.

Epigenetic modifications are involved in gene expressions of tumor-related molecules. Polycomb repressive complex 2 (PRC2) regulates transcription of target genes mainly by trimethylation of $\mathrm{H} 3 \mathrm{~K} 27 \mathrm{me} 3$ (34). Multiple studies have shown that EZH2 is the methylation enzyme subunit of PRC2. Overexpression or mutation of EZH2 can induce 
tumorigenesis and promote tumor progression (35). EZH2 is able to mediate multiple pathological processes in cells, such as cell cycle, cell senescence, and cell differentiation (36).

The present study confirmed the interaction between ST7-AS1 and EZH2 through RIP assay. Subsequently, we speculated whether EZH2 was involved in the malignant progression of GC regulated by ST7-AS1. Notably, the decreased migratory and invasive abilities in GC cells with ST7-AS1 knockdown were further attenuated by EZH2 knockdown. Collectively, ST7-AS1 mediated malignant phenotypes of GC cells via interacting with EZH2.

In conclusion, upregulated ST7-AS1 in GC accelerated proliferative, migratory and invasive abilities, and inhibited apoptosis, thus aggravating the progression of GC. IncRNA ST7-AS1 could be utilized as a promising target for improving clinical outcomes of GC patients.

\section{Acknowledgements}

Not applicable.

\section{Funding}

No funding was received.

\section{Availability of data and materials}

All data generated or analyzed during this study are included in this published article.

\section{Authors' contributions}

SC and FM designed the study and performed the experiments, SC and YW collected the data, FM and PL analyzed the data, SC and FM prepared the manuscript. All authors read and approved the final manuscript.

\section{Ethics approval and consent to participate}

This study was approved by the Ethics Committee of The Fourth Affiliated Hospital of China Medical University (Shenyang, China). Signed informed consents were obtained from the patients and/or guardians.

\section{Patient consent for publication}

Not applicable.

\section{Competing interests}

The authors declare they have no competing interests.

\section{References}

1. Karimi P, Islami F, Anandasabapathy S, Freedman ND and Kamangar F: Gastric cancer: Descriptive epidemiology, risk factors, screening, and prevention. Cancer Epidemiol Biomarkers Prev 23: 700-713, 2014

2. Ding Z, Jiang L, Zhang K and Huang R: Short- and long-term outcomes of conversion in laparoscopic gastrectomy for gastric cancer. J BUON 23: 1004-1012, 2018
3. Xu C, Cui H, Li H, Wu Y, An H and Guo C: Long non-coding RNA ZEB2-AS1 expression is associated with disease progression and predicts outcome in gastric cancer patients. J BUON 24: 663-671, 2019.

4. de Boer WB, Ee H and Kumarasinghe MP: Neoplastic lesions of gastric adenocarcinoma and poximal polyposis syndrome (GAPPS) are gastric phenotype. Am J Surg Pathol 42: 1-8, 2018

5. Markowski AR, Markowska A and Guzinska-Ustymowicz K Pathophysiological and clinical aspects of gastric hyperplastic polyps. World J Gastroenterol 22: 8883-8891, 2016.

6. Nomura $\mathrm{E}$ and Okajima K: Function-preserving gastrectomy for gastric cancer in Japan. World J Gastroenterol 22: 5888-5895, 2016.

7. Akimoto T, Muto S, Kutsuwada T, Kutsuwada K and Nagata D: Peritoneal dialysis and malignancy: An experience with a patient complicated by gastric carcinoma. Clin Med Insights Case Rep 12: 1179547619835176, 2019.

8. Çolak Ş, Gürbulak B, Çakar E and Bektaş H: Resection of mucosal and submucosal gastrointestinal lesions and a double endoscope experience. JSLS 23: e2018.00096, 2019.

9. Huarte M: The emerging role of IncRNAs in cancer. Nat Med 21: 1253-1261, 2015

10. Peng WX, Koirala P and Mo YY: LncRNA-mediated regulation of cell signaling in cancer. Oncogene 36: 5661-5667, 2017.

11. Botti G, Marra L, Malzone MG, Anniciello A, Botti C, Franco R and Cantile M: LncRNA HOTAIR as prognostic circulating marker and potential therapeutic target in patients with tumor diseases. Curr Drug Targets 18: 27-34, 2017.

12. Liu Y, Guo G, Zhong Z, Sun L, Liao L, Wang X, Cao Q and Chen H: Long non-coding RNA FLVCR1-AS1 sponges miR-155 to promote the tumorigenesis of gastric cancer by targeting c-Myc. Am J Transl Res 11: 793-805, 2019.

13. Li J, Xu Q, Wang W and Sun S: MIR100HG: A credible prognostic biomarker and an oncogenic lncRNA in gastric cancer. Biosci Rep 39: BSR20190171, 2019.

14. Marín-Béjar O, Mas AM, González J, Martinez D, Athie A, Morales X, Galduroz M, Raimondi I, Grossi E, Guo S, et al: The human lncRNA LINC-PINT inhibits tumor cell invasion through a highly conserved sequence element. Genome Biol 18: 202, 2017

15. Yamagishi $\mathrm{M}$ and Uchimaru K: Targeting EZH2 in cancer therapy. Curr Opin Oncol 29: 375-381, 2017.

16. Yan KS, Lin CY, Liao TW, Peng CM, Lee SC, Liu YJ, Chan WP and Chou RH: EZH2 in cancer progression and potential application in cancer therapy: A Friend or Foe? Int J Mol Sci 18: 1172, 2017.

17. Wassef M, Luscan A, Aflaki S, Zielinski D, Jansen PW, Baymaz HI, Battistella A, Kersouani C, Servant N, Wallace MR, et al: EZH1/2 function mostly within canonical PRC2 and exhibit proliferation-dependent redundancy that shapes mutational signatures in cancer. Proc Natl Acad Sci USA 116: 6075-6080, 2019.

18. Sun C, Ban Y, Wang K, Sun Y and Zhao Z: SOX5 promotes breast cancer proliferation and invasion by transactivation of EZH2. Oncol Lett 17: 2754-2762, 2019.

19. Labbé DP, Sweeney CJ, Brown M, Galbo P, Rosario S, Wadosky KM, Ku SY, Sjöström M, Alshalalfa M, Erho N, et al: TOP2A and EZH2 provide early detection of an aggressive prostate cancer subgroup. Clin Cancer Res 23: 7072-7083, 2017.

20. Emran AA, Chatterjee A, Rodger EJ, Tiffen JC, Gallagher SJ, Eccles MR and Hersey P: Targeting DNA methylation and EZH2 activity to overcome melanoma resistance to immunotherapy. Trends Immunol 40: 328-344, 2019.

21. Ramakrishnan S, Granger V, Rak M, Hu Q, Attwood K, Aquila L, Krishnan N, Osiecki R, Azabdaftari G, Guru K, et al: Inhibition of EZH2 induces NK cell-mediated differentiation and death in muscle-invasive bladder cancer. Cell Death Differ 26: 2100-2114, 2019.

22. Yu L, Despotovic N, Kovacs MS, Pin CL and Luyt LG: 18F-labeled PET probe targeting enhancer of Zeste homologue 2 (EZH2) for cancer imaging. ACS Med Chem Lett 10: 334-340, 2019.

23. Li Z, Liu ZM and Xu BH: A meta-analysis of the effect of microRNA-34a on the progression and prognosis of gastric cancer. Eur Rev Med Pharmacol Sci 22: 8281-8287, 2018.

24. Lin Y, Zhang CS, Li SJ, Li Z and Sun FB: LncRNA LOC554202 promotes proliferation and migration of gastric cancer cells through regulating p21 and E-cadherin. Eur Rev Med Pharmacol Sci 22: 8690-8697, 2018. 
25. Hernanz N, Rodríguez de Santiago E, Marcos Prieto HM, Jorge Turrión MÁ, Barreiro Alonso E, Rodríguez Escaja C, Jiménez Jurado A, Sierra M, Pérez Valle I, Volpato N, et al: Characteristics and consequences of missed gastric cancer: A multicentric cohort study. Dig Liver Dis 51: 894-900, 2019.

26. Chen C, Tang X, Liu Y, Zhu J and Liu J: Induction/reversal of drug resistance in gastric cancer by non-coding RNAs (Review). Int J Oncol 54: 1511-1524, 2019.

27. Yamagata Y, Saito K, Ban S, Fujii A and Oya M: The origin of p40-negative and CDX2-positive primary squamous cell carcinoma of the stomach: Case report. World J Surg Oncol 17: 53, 2019.

28. Jain VS, Kawale D, Jain SM, Waghmare C and Pemmaraju G: Various addiction patterns, dietary habits, associated medical problems, and socioeconomic status in gastrointestinal malignancies: A prospective study in rural area of Maharashtra, India. J Cancer Res Ther 15: 104-107, 2019.

29. Jarroux J, Morillon A and Pinskaya M: History, discovery, and classification of lncRNAs. Adv Exp Med Biol 1008: 1-46, 2017.

30. Andersen RE and Lim DA: Forging our understanding of lncRNAs in the brain. Cell Tissue Res 371: 55-71, 2018.

31. Léveillé N and Baglio SR: Exosome-transferred lncRNAs at the core of cancer bone lesions. Crit Rev Oncol Hematol 139: $125-127,2019$

32. Mao Z, Wu Y, Zhou J and Xing C: Salinomycin reduces epithelial-mesenchymal transition-mediated multidrug resistance by modifying long noncoding RNA HOTTIP expression in gastric cancer cells. Anticancer Drugs 30: 892-899, 2019.
33. Qin $\mathrm{H}, \mathrm{Xu}$ J, Gong L, Jiang B and Zhao W: The long noncoding RNA ST7-AS1 promotes laryngeal squamous cell carcinoma by stabilizing CARM1. Biochem Biophys Res Commun 512: 34-40, 2019.

34. Huang M, Hou J, Wang Y, Xie M, Wei C, Nie F, Wang Z and Sun M: Long noncoding RNA LINC00673 is activated by SP1 and exerts oncogenic properties by interacting with LSD1 and EZH2 in gastric cancer. Mol Ther 25: 1014-1026, 2017.

35. Chen DL, Ju HQ, Lu YX, Chen LZ, Zeng ZL, Zhang DS, Luo HY, Wang F, Qiu MZ, Wang DS, et al: Long non-coding RNA XIST regulates gastric cancer progression by acting as a molecular sponge of miR-101 to modulate EZH2 expression. J Exp Clin Cancer Res 35: 142, 2016.

36. Yang Y,Zhu F, Wang Q, Ding Y, Ying R and Zeng L: Inhibition of EZH2 and EGFR produces a synergistic effect on cell apoptosis by increasing autophagy in gastric cancer cells. Onco Targets Ther 11: 8455-8463, 2018.

(i) (9) This work is licensed under a Creative Common Attribution-NonCommercial-NoDerivatives 4.0 International (CC BY-NC-ND 4.0) License. 